\title{
Chimeric Fibril-reactive Monoclonal Antibody CAEL-101
}

National Cancer Institute

\section{Source}

National Cancer Institute. Chimeric Fibril-reactive Monoclonal Antibody CAEL-101. NCI

Thesaurus. Code C117241.

A chimeric monoclonal antibody specifically targeting human immunoglobulin light chain (LC)-related fibrils, which may potentially be used in the treatment of light chainassociated (AL) amyloidosis. Upon administration, chimeric fibril-reactive monoclonal antibody CAEL-101 targets and binds to the amyloid-related, conformational epitope on LC-related fibrils. This inhibits fibrillogenesis, induces an Fc-mediated cellular inflammatory response, increases degradation and elimination of AL amyloidomas, and prevents systemic LC-associated amyloid deposits. In AL amyloidosis the amyloid fibrils are composed of immunog lobulin light chain fragments. 